\title{
Nucleon induced recoil ions in microelectronics
}

\author{
Frédéric Wrobel \\ LPES-CRESA, University of Nice, Sophia-Antipolis, France
}

\begin{abstract}
Nucleon induced nuclear reactions in microelectronic devices is a real concern for the radiation community. These nuclear reactions can provoke failures in critical applications in planes and satellites. Nuclear codes are very powerful tools which are required to calculate the number of failures of a given device in a given environment. We present the MC-RED code which is dedicated to the Monte Carlo description of nuclear reactions in microelectronic devices. We also show how its results can be use to calculate the failure rate.
\end{abstract}

\section{Introduction}

Neutrons and protons, which are naturally present in atmosphere [1,2] and space [3] respectively, are able to induce nuclear reactions in microelectronics devices [4]. These reactions lead to the production of secondary ions, which ionize the matter. The produced electron-hole pairs are then likely to modify the behaviour of the device and various failure kinds can occur [5-9]. Nuclear codes are needed to determine the energy deposited in a given microcircuit. Then it is possible to predict the reliability of a given electronic device in a given environment. We present the MC-RED (Monte Carlo Recoil Energy Determination) code dedicated to the nuclear reactions induced in microelectronics. This Monte Carlo code provides information about secondary ions produced during nuclear reactions, and especially about the heavy recoiling ion which is known to have the main effect on an electronic device.

\section{Nucleons induced nuclear reactions in electronic devices}

Two main kinds of device failures can occur, namely the dose effect and the single event effect. As far as the dose effect is concerned, a large amount of ionizing particles contribute all together to the failure. On the contrary, when a single incident particle is involved, the effect is called Single Event Effect (SEE).

Various types of Single Event Effects have been reported. For instance, the Single Event Upset (SEU) denotes a soft failure which upsets a bit memory from a state to another $[4,8]$. The failure is called "soft" because the bit can be restored. By contrast the Single Hard Error [10] may cause permanent device damage, which is then called "hard".

Whatever the kind of SEE occurring in the device (with a nucleon), a nuclear reaction is at play. The occurrence of the SEE is strongly linked to the nature and the energy of the secondary ions produced during this nuclear reaction. Generally, when a nuclear reaction occurs, some light particles are emitted (neutron, proton, alpha...) and one heavy ion remains with a recoil energy. The recoiling ion, which is the most charged among the secondary ions, deposits the highest amount of its energy in the device. Consequently, recoiling ions play a key role in nucleons induced failure in components and the determination of their characteristics is a crucial issue.

\section{Use of nuclear codes}

The knowledge of the nuclear reactions is necessary if one has to deal with the effect of nucleons on components. In this respect, the nuclear codes are very powerful tools. In a first approximation, it is possible to only consider nuclear reactions with Silicon since it is the most abundant material in an electronic device. Nevertheless, notice that recent studies had shown that other elements in the device can have an incidence on the SEU cross section [11-14].

In order to study these nuclear reactions it is useful to distinguish the elastic reactions from the non elastic ones because they are treated in different ways.

The ECIS code [15] provides all the needed information about the elastic reactions and one can easily obtain the number of electron-hole pairs generated along the recoil track in a given device. As regards non elastic reactions, ECIS only gives the cross section and does not allow defining neither the kind of secondary ions produced nor their energies. A code dedicated to the non elastic reactions is then needed.

A possible approach to treat the non-elastic reactions is the Monte Carlo method. Yet, at each stage of the reaction the recoil nucleus is entirely defined, especially by its direction and by its energy. Some Monte Carlo codes like MCNP [16] could give such information but have not been written to do so and are mainly dedicated to the transport of the incident neutron where the recoiling ion is of no interest.

Based on the Monte Carlo approach, various codes, specifically devoted to nuclear reactions in microcircuits have been developed. For instance, we developed the MCRED code [17] in order to treat the recoil energy at low neutron energy that is from $100 \mathrm{keV}$ up to $200 \mathrm{MeV}$. For failures concern this energy range has a great importance since the atmospheric neutron spectrum is rapidly decreasing. The code focuses on all secondary ions and gives their nature, their energy and their direction.

An overview of the MC-RED code is given in figure 1. Reactions are treated in two steps: $i$ ) the preequilibrium by 


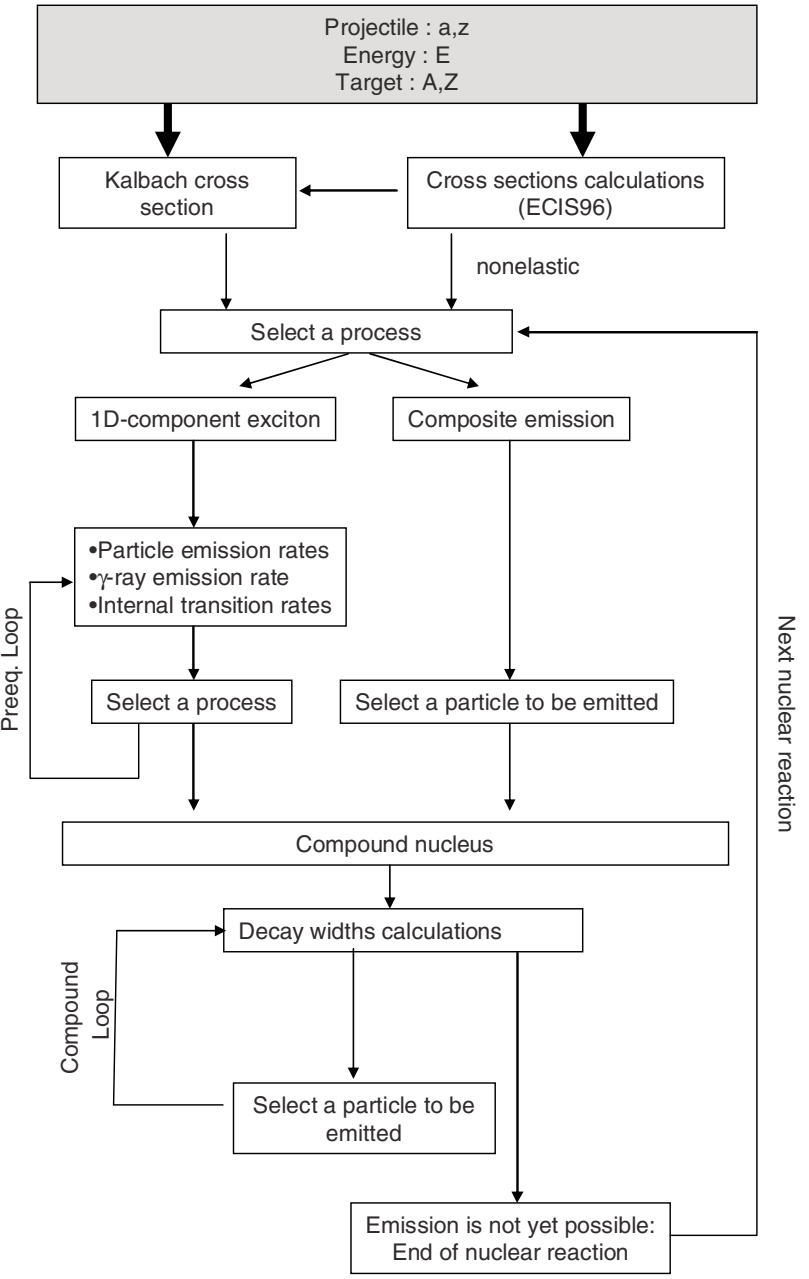

Fig. 1. Overview of the MC-RED nuclear code.

using the 1D-exciton model [18] and the phenomenological parameterisation of Kalbach (pickup, stripping and knockout) [19]; and ii) the equilibrium by using the Hauser-Feshbach theory [20]. These models require the knowledge of cross sections and transmission coefficients. For neutrons and protons, they are obtained by using ECIS96 as a subroutine. To run ECIS96, optical model parameters are extracted either from the parameterisation of local optical parameters from Koning [21] or from the global parameterisation of Koning and Delaroche [21]. For deuterons, tritons, helium-3 and alpha particles, optical parameters are built from those of neutrons and protons by using the folding approach of Watanabe $[22,23]$. Gamma transmission coefficients are linked to the strength functions, which are calculated within the framework of the Generalized Fermi Liquid model (GFL) [24].

The Hauser-Fesbach theory also requires the nuclear level densities. In MC-RED they are calculated by using the GilbertCameron approach [25].

MC-RED allows to built an history of the nuclear reactions with the nature, the energy and the direction of each outgoing secondary ion. Then, this history may be treated with an adapted code whose role is to state, for each nuclear reaction, whether the criterion to trigger a failure is fulfilled or not.
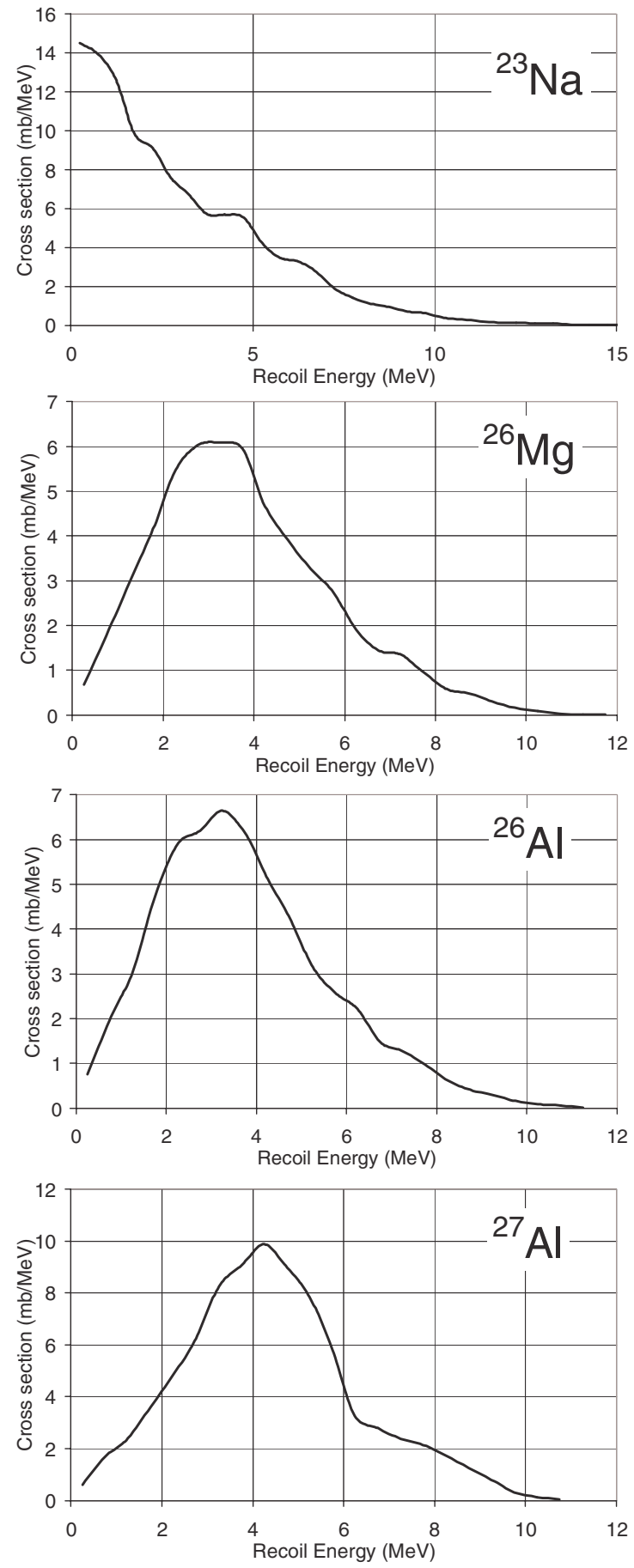

Fig. 2. Examples of secondary ions spectra for the nuclear reaction $\mathrm{n}+{ }^{28} \mathrm{Si}$ at $100 \mathrm{MeV}$.

As an example of results obtained with MC-RED, we have plotted in figure 2 the energy spectra of some secondary ions involved in the neutron-silicon reaction at $100 \mathrm{MeV}$. This kind of results is essential for the prediction tools since the heavy recoiling ion has the highest stopping power and deposit then the highest amount of energy. Nevertheless, modern technologies are more and more sensitive and even light ions such as $\alpha$-particles are able to trigger upsets in a modern technology. This issue is all the more crucial since $\alpha$-particles are widely produced during nuclear reactions. 


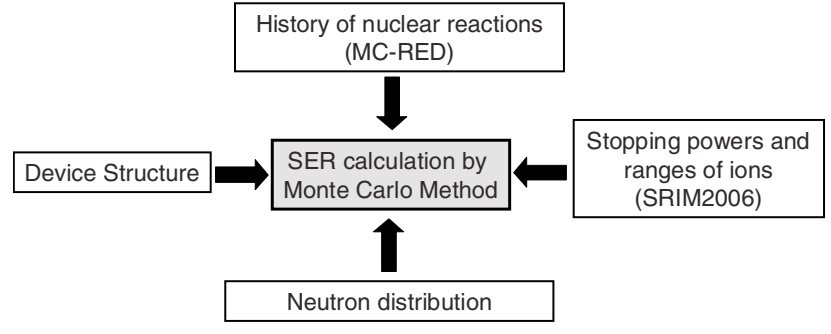

Fig. 3. SER calculation method overview.

Table 1. Applications to some technologies.

\begin{tabular}{lccc}
\hline $\begin{array}{l}\text { Gate } \\
\text { length } \\
(\mathrm{nm})\end{array}$ & $\begin{array}{c}\text { Sensitive } \\
\text { volume } \\
\left(\mu \mathrm{m}^{3}\right)\end{array}$ & $\begin{array}{c}\text { Critical } \\
\text { charge } \\
(\mathrm{fC})\end{array}$ & $\begin{array}{c}\text { Calculated } \\
\text { SER } \\
(/ \mathrm{bit} / \mathrm{h})\end{array}$ \\
\hline 250 & 0.245 & 8 & $1.22 \times 10^{-10}$ \\
130 & 0.025 & 2.5 & $4.69 \times 10^{-11}$ \\
90 & 0.01 & 1.2 & $4.54 \times 10^{-11}$ \\
65 & 0.0035 & 0.8 & $2.44 \times 10^{-11}$ \\
\hline
\end{tabular}

\section{Single Event Upset cross section calculations}

One crucial issue for SEU cross section calculations is the way a nuclear reaction is able to trigger a failure. A common criterion is to assume that each bit of a memory has a region which is sensitive to ionization. In other words, a bit is upset if at least the critical energy is deposited in a sensitive volume. Both the critical energy and the sensitive volume sizes depend on the technology and the kind of memory and can be determined with the help of device simulations tools.

Once these parameters are known, it is possible to calculate the SEU cross section associated to a given device. To do so, nuclear reactions are triggered by the Monte Carlo method in the device. The directions and the energy of the secondary ions are known and one can determine whether a secondary ion crosses a sensitive volume or not. If yes, the energy deposited in the sensitive volume is calculated and compared to the critical one. Finally, if $N_{S E U}$ nuclear reactions have upset a bit while $N_{\text {tot }}$ nuclear reactions have been triggered in the device, the SEU cross section may be written as follows:

$$
\sigma_{S E U}\left(E_{n}\right)=\sigma_{n+S i}\left(E_{n}\right) \cdot \frac{N_{S E U}}{N_{t o t}} \cdot n_{S i}
$$

where $\sigma_{n+S i}\left(E_{n}\right)$ is the ion cross section production for the neutron-silicon nuclear reaction at a neutron energy $E_{n}, n_{S i}$ is the number of silicon nuclei in the device. Finally, the Soft Error Rate (SER), which is the number of failures in a given environment per time unit and per bit, is given by:

$$
S E R=\int \sigma_{S E U}(E) \frac{d N(E)}{d E} d E,
$$

where $\frac{d N(E)}{d E}$ is the neutron distribution, in the atmosphere for instance. Figure 3 is an overview of the SER calculation with the needed inputs.

Table 1 shows some calculated SER for four technologies at avionic altitude $(12 \mathrm{~km})$. Results are of the same order of magnitude than that observed experimentally. For these devices the SER is typically around $10^{-10} \mathrm{SEU} / \mathrm{bit} / \mathrm{h}$, that is to say that a 1 Go memory should undergo typically one SEU every hour. Actually, every SEU are not critical and some of them may be corrected. Nevertheless, when several SEU occur simultaneously (multiple bits upset), the problem is a major concern.

\section{Conclusions}

Neutrons and protons can provoke failures in microcircuits. The occurrence of these failures can be determined either by experiments or by calculations. Experimentally, it is however difficult to have a good accuracy since a long irradiation time is required. In this work, we have focused on the SER calculations. The starting point is a good knowledge of nuclear reactions that is the nature, the energy and the directions of all secondary ions. This information is obtained with the help of nuclear codes. We have presented the MC-RED code, which was specifically developed for microelectronic concern. It gives a history of nuclear reactions, which can be used as an input of SER prediction tools. The current version of MCRED is based on the composite emission, the 1-D exciton model and the Hauser-Feshbach theory. This kind of code is quite CPU consuming and that is the reason why recent efforts have been made to improve the computational method. We are currently developing the DHORIN code (Detailed History Of Recoil Ions induced by Nucleons), which allows reducing the calculation time. Moreover, the code uses improved models compared to MC-RED, that is 2-D exciton model but also the generalized evaporation model to account for the emission of heavier ions than helium.

Finally, let us emphasize that the Monte Carlo approach gives all the needed information and especially the correlation between secondary ions produced during a nuclear reaction. Some kinds of failures, such as MBU, are yet triggered by several ions in different bits.

\section{References}

1. W.N. Hess, H.W. Patterson, R. Wallace, E.L. Chupp, CosmicRay Neutron Energy Spectrum, Phys. Rev. 116, 445 (1959).

2. W.N. Hess, E.H. Canfield, R.E. Lingenfelter, Cosmic-Ray Neutron Demography, J. Geophys. Res. 66, 665 (1961).

3. J.F. Ziegler, Terrestrial cosmic rays and soft errors, IBM J. Re. Develop., Vol. 40, No. 1 (Jan. 1996), pp. 19-39.

4. E. Normand, Single event upset at ground level, IEEE Trans. Nucl. Sci., Vol. 43 (Dec. 1996), pp. 2742-2750.

5. J.F. Leavy, R.A. Poll, Radiation-Induced integrated Circuit Latchup, IEEE Trans. Nucl. Sci., Vol. NS-16, No. 6 (Dec. 1969), pp. 96-103.

6. T.F. Wrobel, On heavy ion induced hard-errors in dielectric structures, IEEE Trans Nucl. Sci., Vol. 34, (1987), pp. 1262 1268.

7. S. Kuboyama, K. Sugimoto, S. Shugyo, S. Matsuda, T. Hirao, Single-event burnout of epitaxial bipolar transistors, IEEE Transactions Nucl. Sci., Vol. 45, Issue 6, Part 1 (Dec. 1998), pp. 2527-2533.

8. E. Normand, Single Event Upset in Avionics, IEEE Trans. Nucl. Sci., Vol. 40, N² (April 1993). 
9. R.A. Reed, M.A. Carts, P.W. Marshall, C.J. Marshall, O. Musseau, P.J. McNulty, D.R. Roth, S. Buchner, J. Melinger, T. Corbiere, Heavy ion and proton-induced single event multiple upset, IEEE Trans. Nucl. Sci., Vol. 44, Issue 6, Part 1 (Dec. 1997), pp. 2224-2229.

10. J.L. Barth, J.W. Adolphsen, G.B. Gee, Single event effects on commercial SRAMs and power MOSFETs: final results of the CRUX flight experiment on APEX, Radiation Effects Data Workshop, 1998. IEEE, 1998, pp. 1-10.

11. F. Wrobel, J.-M. Palau, M.-C. Calvet, P. Iacconi, Contribution of SiO2 in Neutron-Induced SEU in SRAMs, IEEE Trans. Nucl. Sci., Vol. 50, No. 6 (Dec. 2003), pp. 2055-2059.

12. A.D. Tipton, J.A. Pellish, R.A. Reed, R.D. Schrimpf, R.A. Weller, M.H. Mendenhall, B. Sierawski, A.K. Sutton, R.M. Diestelhorst, G. Espinel, J.D. Cressler, P.W. Marshall, G. Vizkelethy, Multiple-Bit Upset in $130 \mathrm{~nm}$ CMOS Technology, IEEE Trans. Nucl. Sci, Vol. 53, No. 6 (Dec. 2006).

13. C.L. Howe, R.A. Weller, R.A. Reed, M.H. Mendenhall, R.D. Schrimpf, K.M. Warren, D.R. Ball, L.W. Massengill, K.A. LaBel, J.W. Howard, N.F. Haddad, Role of Heavy-Ion Nuclear Reactions in Determining On-Orbit Single Event Error Rates, IEEE Trans. Nucl. Sci., Vol. 52, No. 6 (Dec. 2006), pp. 21822188.
14. A.S. Kobayashi, D.R. Ball, K.M. Warren, R.A. Reed, N. Haddad, M.H. Mendenhall, R.D. Schrimpf, R.A. Weller, The Effect of Metallization Layers on Single Event Susceptibility, IEEE Trans. Nucl. Sci., Vol. 52, No. 6 (Dec. 2006), pp. 2189-2193.

15. J. Raynal, Notes on ECIS 95, CEA Saclay Report No. CEA-N2772, 1994.

16. L.S. Waters, ed., MCNPX User's Manual, Version 2.3.0, LA-UR02-2607 (April 2002)

17. F. Wrobel, J.-M. Palau, P. Iacconi, M.-C. Palau, B. Sagnes, F. Saigné, Methodology to Compute Neutron Induced Alphas Contribution on the SEU Cross Section in Sensitive RAMs, IEEE Trans. Nucl. Sci., Vol. 51, No. 6 (Dec. 2004), pp. 3291-3297.

18. A.J. Koning, M.C. Duijvestijn, Nucl. Phys. A 744, 15 (2004).

19. C. Kalbach, Z. Phys. A 283, 401 (1977).

20. M. Uhl, Acta Physica. Austri. 31, 245 (1970).

21. A.J. Koning, J.P. Delaroche, Nucl. Phys. A 713, 231 (2003).

22. S. Watanabe, Nucl. Phys. 8, 484 (1958).

23. D.G. Madland, in Proceedings of a Specialists' Meeting on Preequilibrium Nuclear Reactions, Austria, Feb. 10-12 (1988), p. 103.

24. S.F. Mughabghab, C.L. Dunford, Phys. Lett. B 487, 155 (2000).

25. A.Gilbert, A.G.W. Cameron, Can. J. Phys. 43, 1446 (1965). 